\title{
Propriedades psicométricas iniciais do Acceptance and Action Questionnaire - II - versão brasileira
}

\author{
Leonardo Martins Barbosa - Universidade de Brasilia, Brasilia, Brasil \\ Sheila Giardini Murta - Universidade de Brasilia, Brasília, Brasil
}

\begin{abstract}
Resumo
O modelo de flexibilidade psicológica (FP) dispõe de evidências em diversos países, mas sua investigação no Brasil é limitada pela ausência de instrumentos adaptados. Esse estudo reúne evidências psicométricas de um instrumento de avaliação da FP, o Acceptance and Action Questionnaire-II, adaptado para o Brasil. Após tradução e adaptação, o instrumento foi respondido por 1.352 estudantes universitários, e parte da amostra também respondeu escalas de resiliência, saúde geral, depressão e ansiedade. Os resultados indicaram estrutura unidimensional e confiabilidade satisfatória. A FP dos homens foi superior à das mulheres e tendeu a aumentar de acordo com a idade. Também houve correlação positiva com resiliência e saúde geral, e negativa com depressão e ansiedade. As propriedades psicométricas foram satisfatórias, mas ainda devem ser avaliadas em amostras diversificadas. O instrumento parece adequado para uso tanto no estudo sobre mudança de comportamento quanto sobre mecanismos de mudança terapêutica.

Palavras-chave: validade do teste; AAQ-II; flexibilidade psicológica; terapia cognitivo-comportamental; psicoterapia.
\end{abstract}

Initial psychometric properties of Acceptance and Action Questionnaire - II - Brazilian version

\begin{abstract}
Psychological flexibility (PF) model has extensive evidence in several countries but its research in Brazil is limited due to lack of suitable instruments. This study gathers psychometric evidence from an FP instrument, Acceptance and Action Questionnaire-II, adapted for Brazil. After translated and adapted, 1,352 college students filled it, and same subsamples also filled scales on resilience, general health, depression and anxiety. Results indicated unidimensional structure and satisfactory reliability. FP in males was superior to females and increased with aging. There was also a positive correlation with resiliency and overall health, and a negative one with depression and anxiety. Psychometric properties were satisfactory, but ought to be evaluated in diverse samples. The instrument seems suitable for use both in studying behavior change and mechanisms of therapeutic change.

Keywords: validation; AAQ-II; psychological flexibility; action and commitment therapy; psychotherapy.
\end{abstract}

Propiedades psicométricas iniciales del Acceptance and Action Questionnaire - II - Versión Brasileña

\begin{abstract}
Resumen
El modelo de flexibilidad psicológica (FP) dispone de evidencias en diversos países, pero su investigación en Brasil es limitada por falta de instrumentos adaptados. Este estudio reúne evidencias psicométricas de un instrumento de evaluación de la FP, el Acceptance and Action Questionnaire-II, adaptado para Brasil. Tras la traducción y adaptación, respondieron al instrumento 1.352 estudiantes universitarios, y parte de la muestra también respondió a escalas de resiliencia, salud general, depresión y ansiedad. Los resultados indicaron estructura unidimensional y confiabilidad satisfactoria. El modelo de FP de los hombres fue superior al de las mujeres y tiende a aumentar con la edad. Hubo correlación positiva con la resiliencia y salud general, y negativa con la depresión y ansiedad. Las propiedades psicométricas fueron satisfactorias, pero aún deben ser evaluadas en muestras diversificadas. El instrumento parece adecuado tanto para el estudio sobre cambios de comportamiento, como para el estudio sobre mecanismos de cambios terapéuticos.

Palabras-clave: validez del test; AAQ-II, flexibilidad psicológica; terapia cognitiva-conductual; psicoterapia.
\end{abstract}

Frequentemente segue-se uma regra arriscada: normal é estar bem e eliminar o mal-estar é a prioridade. Contudo, as tentativas de alcançar o bem-estar podem ser ineficazes (Purdon \& Clark, 2001; Wegner, 1994), pois pensamentos e sentimentos adversos ocorrem mesmo em circunstâncias favoráveis. Paradoxalmente, por outro lado, retirar a ênfase da redução de sintomas pode favorecer a própria redução de sintomas (Hayes, Strosahl, \& Wilson, 2011).

Basear as decisões em valores é uma alternativa a essa regra (Hayes et al., 2011). Quando ações são realizadas dessa maneira, suas consequências representam uma manifestação dos valores, independentemente de quanto desconforto está associado. No entanto, é difícil mensurar o quanto as decisões são guiadas por princípios, e não pela evitação do sofrimento. Por isso, o objetivo deste estudo é apresentar as evidências psicométricas iniciais da versão brasileira de um instrumento de avaliação da capacidade de agir com base em valores. Essa capacidade é denominada Flexibilidade psicológica (FP), a "habilidade de contatar o momento presente de forma mais completa, como um ser humano consciente, e mudar ou persistir no comportamento quando isso servir aos fins valorizados" (Hayes et al., 2006, p. 7). 


\section{Flexibilidade psicológica e terapia de aceitação e compromisso}

O construto flexibilidade psicológica foi proposto pela terapia de aceitação e compromisso (ACT; Hayes et al., 1999, 2011), uma abordagem cognitivo-comportamental. A ACT se baseia em uma filosofia funcionalista (Hayes, 1993; Pepper, 1966), em uma estratégia abrangente de desenvolvimento científico, a ciência contextual comportamental ada a maior desist (Hayes Wilson, \& Barnes-Holmes, 2012), e na teoria dos quadros relacionais (Hayes et al., 2001), uma abordagem comportamental pós-skinneriana da cognição e da linguagem. A ACT é parte das terapias cognitivo-comportamentais contextuais, que priorizam a alteração do contexto, e não do conteúdo, de pensamentos, sentimentos e sensações (Hayes et al., 2011).

Além de constituir uma abordagem terapêutica, a ACT propõe uma abordagem de saúde mental que integra saúde e psicopatologia (Hayes, 2006), constituindo um modelo unificado de funcionamento humano (Hayes et al., 2011). Esse modelo de funcionamento psicológico é representado pelo conceito de flexibilidade psicológica e pelo seu inverso, a inflexibilidade psicológica. O funcionamento saudável resulta da interação entre processos - aceitação, defusão, atenção ao momento presente, self observador, valores e comprometimento - que favorecem a disponibilidade em se adaptar às circunstâncias da vida, promovendo FP. O funcionamento psicopatológico, por outro lado, deriva da interação de processos de indisponibilidade para adaptação - evitação, fusão, atenção rígida, self conceitualizado, valores pouco claros e inação ou impulsividade -, promovendo inflexibilidade psicológica e disfuncionalidade.

Inicialmente, esse modelo era denominado evitação experiencial (EE), construto definido como o "fenômeno que ocorre quando uma pessoa está indisposta a permanecer em contato com experiências privadas particulares (...) e age para alterar a forma ou a frequência desses eventos e dos contextos que os ocasionam" (Hayes et al., 1996, p. 1154). A EE, um fenômeno tão abrangente que pode ser considerada uma categoria diagnóstica funcional, subjaz transtornos como o obsessivo-compulsivo, o estresse agudo, o de estresse pós-traumático, a fobia social e o pânico (Salters-Pedneault, Tull, \& Roemer, 2004).

A transição para a FP representou a adoção de um modelo mais amplo. $\mathrm{O}$ novo construto abarca a ampla capacidade de adaptação direcionada para a vivência dos valores, que pode ser prejudicada não apenas por comportamentos evitativos. Apesar dessa distinção, os conceitos ainda são considerados como equivalentes (Bond et al., 2011). Além disso, a FP se sobrepõe a construtos mais tradicionais, como resiliência, conscienciosidade, autorregulação e tolerância ao sofrimento (Kashdan \& Rottenberg, 2010). Em meio a essa variedade, a principal contribuição da FP é estabelecer um novo parâmetro de avaliação - os valores pessoais, deslocando a ênfase na redução de sintomas para a preservação e melhoria da capacidade de funcionamento (Gloster, Klotsche, Chaker, Hummel, \& Hoyer, 2011).

A inflexibilidade psicológica, processo oposto à FP, é caracterizada pela inabilidade em mudar ou persistir em comportamentos orientados por valores e está associada à disfuncionalidade. Os prejuízos variam de tentativas inócuas de regular eventos internos a estilos de vida orientados pelo medo de sofrer (Hayes \& Gifford, 1997; Hayes et al., 1996). Independentemente do contexto, a não realização dos valores pessoais é o efeito comum.

As evidências dos prejuízos associados à inflexibilidade psicológica em contextos variados são amplas. Revisões de literatura mostram a associação com diversos quadros disfuncionais, como uso de drogas, estresse pós-traumático, sequelas do abuso sexual e tricotilomania (Chawla \& Ostafin, 2007); depressão e transtornos de ansiedade (Kashdan \& Rottenberg, 2010; Salters-Pedneault et al., 2004); dor crônica, aprendizagem e desempenho no trabalho, qualidade de vida e gravidade dos sintomas do transtorno de personalidade borderline (Ruiz, 2010); ajustamento emocional e absenteísmo, após um período de 12 meses (Bond et al., 2011).

O funcionamento cotidiano, um dos principais parâmetros para a mensuração do construto, é comumente prejudicado. A inflexibilidade psicológica é um preditor da redução de experiências afetivas positivas e aumento das negativas (Kashdan, Barrios, Forsyth, \& Steger, 2006); neuroticismo, prejuízos em atividades rotineiras, redução de tempo livre e de contatos sociais (Gloster et al., 2011). Em crianças, está associada ao estilo parental autoritário (Williams, Ciarrochi, \& Heaven, 2012). No contexto organizacional, níveis de FP predizem desempenho (Hayes et al., 2006), absenteísmo e sofrimento psicológico (Bond et al., 2011)

Os dados sugerem que a FP contribui para o funcionamento humano em diferentes contextos (Hayes et al., 2006). Uma análise adequada para checar essa contribuição é o teste de mediação, que avalia se as mudanças na variável dependente decorrem de alterações nas variáveis dependente e mediadora, e se a 
variável mediadora é a responsável pelo efeito da variável independente (Baron \& Kenny, 1986).

A literatura sobre processos terapêuticos caracteriza os mediadores como mecanismos de mudança, fatores efetivamente responsáveis pela mudança terapêutica do comportamento (Barlow et al., 2013; Kazdin, 2007). A identificação desses mecanismos é uma antiga demanda da pesquisa sobre avaliação do processo terapêutico (Paul, 1967): existem centenas de tratamentos (Kazdin, 2000), uma abundância artificial baseada na reapresentação dos mesmos princípios terapêuticos em métodos aparentemente distintos (Kazdin, 2007).

Os mecanismos, no entanto, não se restringem ao contexto clínico: são elementos básicos do funcionamento humano. Uma revisão indica, por exemplo, que a FP medeia relações entre abuso sexual infantil e sofrimento psicológico atual, tratamento do tabagismo e da obesidade, autocuidado de pacientes com diabetes, redução do número de convulsões e aumento da qualidade de vida de portadores de epilepsia, redução do preconceito e adaptação funcional de pacientes com dor crônica (Ciarrochi, Bilich, \& Godsell, 2010). Além disso, há evidências de mediação da FP das relações entre enfrentamento, estilos de resposta e traços de ansiedade, assim como entre regulação emocional e número de experiências cotidianas positivas e negativas (Kashdan et al., 2006).

Histórico e limitaçoes do Acceptance and Action Questionnaire-II (AAQ-II)

Hayes et al. (2004) elaboraram uma primeira medida do construto, nomeada de Acceptance and Action Questionnaire-I (AAQ-I), com o objetivo de ampliar a pesquisa sobre evitação experiencial. A partir do AAQ-I, foram desenvolvidas versões para avaliação da FP em diversas condições específicas, coerentemente com a natureza contextual do construto. Há escalas para uso em casos de dor crônica, tabagismo, manejo da diabetes, dor de ouvido, obesidade, epilepsia e enfrentamento de sintomas psicóticos, entre outros (Bond et al., 2011). Essas escalas específicas são mais sensíveis à mudança, aumentando sua relevância clínica (Hayes et al., 2004).

O desenvolvimento do AAQ-I gerou um forte estímulo à pesquisa sobre FP, mas apresentava duas limitações importantes: itens de difícil compreensão e baixa consistência interna. Por isso, Bond et al. (2011) desenvolveram a segunda versão do instrumento, o AAQ-II. Nesse estudo, o construto-alvo foi denominado "variadamente como aceitação, evitação experiencial ou inflexibilidade psicológica" (Bond et al., 2011, p. 676). Essa ampla delimitação mostra que o conceito ainda está em desenvolvimento.

O AAQ-II foi aplicado a 2.816 participantes dos Estados Unidos e Reino Unido, em conjunto com outras nove medidas gerais de saúde mental (p. ex., depressão, qualidade de vida e satisfação no trabalho) para verificar propriedades de validade concorrente, convergente, preditiva, discriminante e incremental. A versão inicial continha 49 itens, gerados por um grupo de especialistas em ACT, e a versão final foi composta por sete itens sobre comportamentos inflexíveis, com cargas variando entre 0,59 e 0,82 . Os resultados confirmaram um único fator, de acordo com a teoria, explicando $50,68 \%$ da variância total. A correlação entre as duas versões do AAQ foi de 0,97, e as correlações entre o AAQ-II e as outras medidas variaram entre 0,25 e 0,71 . A confiabilidade média, um dos principais problemas da versão anterior $(\alpha=0,70)$, aumentou para 0,84 .

O AAQ, também, passou por processo de verificação das evidências de validade em outros países, como Portugal (Pinto-Gouveia \& Gregório, 2007), Espanha (Ruiz, Herrera, Luciano, Cangas, \& Beltrán, 2013) e Holanda (Boelen \& Reijntjes, 2008). Apesar das amplas evidências de consistência da FP como modelo de funcionamento e da sua aplicabilidade, ainda não há instrumentos desenvolvidos ou adaptados para o Brasil. Por isso, o presente estudo consistiu em traduzir e adaptar o AAQ-II para o contexto brasileiro e submeter os dados à verificação das evidências de validade de construto via análise fatorial exploratória.

A pesquisa sobre FP apresenta limitações importantes. A natureza comportamental do construto cria um problema: FP é uma categoria de ações contextualizadas, e não uma variável psicológica hipotética, violando um pressuposto básico de algumas análises estatísticas. Além disso, a FP é definida em função do contexto, o que dificulta sua mensuração apenas com instrumentos gerais de autorrelato (Gloster et al., 2011; Hayes et al., 2004; Kashdan \& Rottenberg, 2010). A natureza abrangente do fenômeno é outro obstáculo à mensuração: nenhuma das escalas disponíveis, efetivamente, avalia FP, apenas seus processos correlatos (Ciarrochi et al., 2010). As evidências de validade de construto, discriminativa e incremental da FP, assim como seu papel mediador na mudança terapêutica, já foram apontadas como limitações (Chawla \& Ostafin, 2007; Hofmann \& Asmundson, 2008); mas há evidências consistentes sobre sua unidade e validade (Gloster 
et al., 2011) e poder mediador (Hayes et al., 2006; Ruiz, 2010). Apesar do grande potencial, o conceito é recente e, ainda, precisa ser explorado em diferentes contextos, como escolas e organizações.

\section{Método}

\section{Participantes}

O AAQ-II - Versão Brasileira (Anexo 1) foi respondido por 1.352 estudantes universitários. A coleta de dados para o processo de verificação das evidências de validade de construto envolveu 834 participantes de oito cidades: Aracaju/SE ( $n=44$ / 5,3\%), Belém/ PA $(n=141 / 17 \%)$, Brasília/DF $(n=147-17,6 \%)$, Goiânia/GO ( $n=94-11,2 \%)$, Rio Branco/AC $(n=116$ - 14\%), Rio de Janeiro/RJ ( $n=123$ - 14,7\%), Unaí/MG $(n=141-17 \%)$ e São Paulo/SP $(n=27-3,2 \%)$. A coleta para comparar a FP com escores de outros instrumentos envolveu 518 participantes da cidade de Brasília. A amostra foi composta por $65,3 \%$ de mulheres e apresentou média de 24,71 anos de idade $(D P=7,83)$.

\section{Instrumentos}

O AAQ-II original foi traduzido para o português por quatro pesquisadores independentes, dois especialistas em psicologia social e dois especialistas em psicologia clínica. As versões foram comparadas por dois juízes especialistas em psicologia clínica, que fizeram modificações para obter uma tradução mais concisa e clara. Por exemplo, três pesquisadores criticaram o uso conjunto das palavras "preocupo" e "preocupações" no item 3, mas esses termos foram mantidos para preservar a semelhança com a versão original; no item 6 , dois pesquisadores sugeriram o uso de "me parece" ou "parece-me", mas o termo "parece" foi mantido por ser mais utilizado na linguagem cotidiana.

A versão adaptada foi submetida à verificação das evidências de validade semântica por meio da análise de dois grupos de quatro estudantes universitários (Pasquali, 2010), mantendo-se todos os sete itens (Tabela 2). As respostas são dadas de acordo com uma escala de 7 pontos, entre 1 (nunca) e 7 (sempre), e os escores finais variam entre 7 e 49 . Os itens, originalmente, avaliam inflexibilidade psicológica, mas as respostas foram invertidas para facilitar a compreensão dos resultados. Assim, escores mais elevados indicam maior flexibilidade psicológica.

A Escala de Resiliência Connor-Davidson-10 (CD-RISC 10; Lopes \& Martins, 2011) é utilizada para avaliação da resiliência. É composta por 10 itens, como "Dar a volta por cima", e apresenta estrutura unifatorial $(\alpha=0,82)$. Os itens são respondidos em uma escala Likert de 5 pontos, entre 0 (nunca é verdade) e 4 (sempre é verdade), com escores variando entre 0 e 40 pontos.

A Center for Epidemiological Studies - Depression (CES-D; Batistoni, Neri, \& Cupertino, 2007) é utilizada para avaliação da depressão. A escala é composta por 20 itens $(\alpha=0,86)$ que avaliam 4 fatores: afetos negativos (p.ex., "Senti-me sozinho"), afetos positivos (p.ex., "Aproveitei minha vida") e dificuldades de iniciar comportamentos (p.ex., "Falei menos do que o habitual"), além de um quarto fator não interpretado (p.ex., "Meu sono não foi repousante"), avaliados por meio de uma escala Likert de 4 pontos, entre 1 (raramente) e 4 (sempre), com escores variando entre 20 e 80.

O Inventário de Ansiedade Traço-Estado - Traço (IDATE-T; Fioravanti et al., 2006) é utilizado para avaliação da ansiedade. É composto por 20 itens $(\alpha=0,88)$, respondidos em uma escala de 1 (quase nunca) a 4 (quase sempre) sobre como o respondente geralmente de sente, com escores variando entre 20 e 80 . A estrutura é bifatorial, indicando presença ou ausência de ansiedade.

O Questionário de Saúde Geral - 12 (QSG-12; Gouveia et al., 2003), avalia a saúde mental geral. É composto de 12 itens que avaliam dois fatores: depressão $(\alpha=0,81)$ e ansiedade $(\alpha=0,66)$. Os escores finais variam entre 12 e 48 , com itens respondidos de acordo com escalas de 1 (absolutamente não) a 4 (muito mais que de costume) para itens negativos, e de 1 (mais que de costume) a 4 (muito menos que de costume) para itens positivos.

\section{Procedimentos}

Inicialmente, o projeto deste estudo foi submetido a um Comitê de Ética em Pesquisa. Após a aprovação, diretores e docentes de instituições de ensino superior foram contatados para autorizar a aplicação dos questionários em estudantes universitários. Todos os participantes consentiram com a participação no estudo e preencheram os instrumentos em sala de aula, durante o horário letivo regular, em sessão única. Nas aplicações apenas com o AAQ-II, o tempo de preenchimento foi inferior a 5 minutos. Nas aplicações para comparação com escores de outros instrumentos, o tempo de preenchimento foi de aproximadamente 25 minutos, sendo que os instrumentos foram aplicados em diferentes sequências, e não foram verificados efeitos da ordem de preenchimento. 


\section{Análise dos dados}

A fatorabilidade da escala foi verificada por meio do teste de adequação da amostra Kaiser-Meyer-Olkin (KMO), e o número de fatores foi avaliado por meio das análises de componentes principais (PC) e paralela (PA). Em seguida, foi realizada a análise fatorial exploratória (PAF) e calculados os alfas de Cronbach. Por fim, os escores do AAQ-II foram correlacionados por meio do coeficiente de correlação produto-momento de Pearson, aos construtos de saúde geral, resiliência, depressão e ansiedade, para verificar sua semelhança com esses fenômenos.

\section{Resultados}

As estatísticas descritivas são apresentadas na Tabela 1. A amostra utilizada para verificar as evidências de validade de construto do AAQ-II foi coletada em diferentes cidades, por isso foi maior do que as amostras utilizadas para avaliar as convergência e divergência do AAQ-II com outros construtos, coletadas apenas em Brasília. A proporção de homens superou a de mulheres apenas na amostra em que foram aplicadas as escalas de resiliência (CD-RISC) e depressão (CES-D). A média de idade variou entre 20 e 31 anos, indicando representantes jovens, o que pode ser justificado pela sua composição exclusiva de estudantes universitários. Por fim, são apresentados escores médios e desvios-padrão dos cinco instrumentos aplicados.

As análises apresentaram resultados satisfatórios, como mostra a Tabela 2. A matriz apresentou boa fatorabilidade $(\mathrm{KMO}=0,87)$, e a $\mathrm{PC}$ mostrou que apenas o autovalor empírico para um fator único $(\lambda=3,9)$ superava o autovalor aleatório correspondente indicado pela PA $(\lambda=1,13)$. O fator único extraído pela PAF apresentou cargas fatoriais acima de $0,3 \mathrm{em}$ todos os itens (0,61 a 0,75), explicando 48,4\% da variância. O índice de consistência interna foi 0,86 . A FP média foi 32,54 $(D P=8,62)$, com uma associação positiva entre idade e flexibilidade $(r=0,13 ; p<0,001)$, mostrando uma tendência suave ao aumento da flexibilidade com o passar dos anos. Homens apresentaram flexibilidade média

Tabela 1. Estatísticas Descritivas dos Construtos Avaliados

\begin{tabular}{lcccccc}
\hline Instrumento & $\mathrm{n}$ & Sexo feminino & $\begin{array}{c}\text { Idade } \\
(\mathrm{M})\end{array}$ & $\begin{array}{c}\text { Idade } \\
(\mathrm{DP})\end{array}$ & $\begin{array}{c}\text { Escore } \\
(\mathrm{M})\end{array}$ & $\begin{array}{c}\text { Escore } \\
(\mathrm{DP})\end{array}$ \\
\hline AAQ-II & 834 & $65,3 \%$ & 24,71 & 7,83 & 32,54 & 8,62 \\
CD-RISC 10 & 179 & $42,5 \%$ & 30,73 & 9,06 & 28,23 & 5,96 \\
CES-D & 179 & $42,5 \%$ & 30,73 & 9,06 & 35,83 & 8,53 \\
IDATE-T & 210 & $73,8 \%$ & 25,1 & 7,52 & 43,38 & 10,54 \\
QSG-12 & 129 & $65,8 \%$ & 20,61 & 4,17 & 30,59 & 3,7 \\
\hline
\end{tabular}

Tabela 2. Análise Fatorial Exploratória do AAQ-II - Versão Brasileira

\begin{tabular}{lc}
\hline item & carga fatorial \\
\hline 1. minhas experiências e lembranças dolorosas dificultam que eu viva a vida que eu gostaria. & 0,73 \\
2. tenho medo dos meus sentimentos. & 0,61 \\
3. eu me preocupo em não conseguir controlar minhas preocupações e sentimentos. & 0,64 \\
4. minhas lembranças dolorosas me impedem de ter uma vida plena. & 0,75 \\
5. emoções causam problemas na minha vida. & 0,70 \\
6. parece que a maioria das pessoas lida com suas vidas melhor do que eu. & 0,71 \\
7. preocupações atrapalham o meu sucesso. & 0,72 \\
\hline variância total explicada & $48,42 \%$ \\
média da escala & 32,54 \\
desvio-padrão da escala & 8,62 \\
alfa de Cronbach & 0,87 \\
\hline
\end{tabular}


$[M=34,97,95 \%$ IC $(33,9 ; 36,05), D P=8,39]$ superior às mulheres $[M=31,43,95 \%(30,71 ; 32,15), D P=8,49]$, $t(769)=5,32, p<0,001, d=0,42$.

A Tabela 3 apresenta as correlações entre a FP e construtos indicativos de saúde mental. Os escores do AAQ-II apresentaram correlações positivas com os instrumentos de saúde geral $(r=0,21)$ e resiliência $(r=0,35)$ e correlações negativas com os resultados de depressão $(r=-0,62)$ e ansiedade $(r=-0,71)$. Foram realizadas correlações parciais entre as medidas e todos os valores se mantiveram significativos.

\section{Discussão}

O processo de verificação das evidências de validade do AAQ-II mostrou que o instrumento apresenta propriedades psicométricas satisfatórias. A análise fatorial exploratória indicou uma estrutura unifatorial, que explica 48,42\% da variância do construto. Esse resultado corresponde à teoria: diferentes processos psicológicos, em interação, promovem a flexibilidade psicológica. $\mathrm{Da}$ mesma forma, embora os itens da escala avaliem esses processos correlatos, mas configuraram um único fator. As cargas fatoriais foram elevadas e o valor, também, alto do alpha de Cronbach de 0,87 sugere a confiabilidade do instrumento. Esses resultados são equivalentes ou superiores aos encontrados nas versões validadas em outros países, como Espanha ( $\alpha$ entre 0,75 e 0,93; Ruiz, Herrera, Luciano, Cangas, \& Beltrán, 2013), Estados Unidos/Reino Unido ( $\alpha$ médio $=0,84$; Bond et al., 2011), Holanda ( $\alpha=0,74$; Boelen \& Reijntjes, 2008), Itália $(\alpha=0,83$; Pennato, Berrocal, Bernini, \& Rivas, 2013) e Portugal ( $\alpha=0,84$; Pinto-Gouveia \& Gregório, 2007).

As correlações com processos associados à saúde mental também se mostraram consistentes com o modelo teórico. Correlações elevadas foram encontradas com construtos que sinalizam psicopatologia - depressão e ansiedade, como indicado em revisões anteriores (Kashdan \& Rottenberg, 2010;
Salters-Pedneault et al., 2004). Por outro lado, os construtos relacionados ao funcionamento saudável apresentaram correlações positivas, mas pequenas, com os escores de FP. Este último resultado é coerente com a literatura (Kashdan \& Rottenberg, 2010), que sugere a possibilidade de integrar no conceito de FP a pesquisa de fenômenos tradicionalmente estudados de forma assistemática, como resiliência, autorregulação e tolerância ao sofrimento. As correlações obtidas para depressão, ansiedade e resiliência são equivalentes às encontradas em outras investigações das propriedades psicométricas do AAQ (Bond et al., 2011; Hayes et al., 2004), valores considerados como evidências de validade convergente pelos autores. No entanto, o método utilizado neste estudo impede conclusões seguras sobre propriedades de convergência ou divergência com outros instrumentos.

Ao longo da história da psicologia clínica, diversos pesquisadores (Kazdin, 2007; Paul, 1967; Rosenzweig, 1936) apontaram a necessidade de identificar os mecanismos responsáveis pela mudança terapêutica. Até o momento, as evidências mostram que a FP pode ter esse papel (Kashdan \& Rottenberg, 2010), sendo que baixos escores de flexibilidade representam um traço generalizado de vulnerabilidade psicológica (Kashdan et al., 2006). Assim, a possibilidade da avaliação da FP em contexto nacional contribui não apenas para identificar riscos relativos a diversos transtornos emocionais, mas também para o possível desenvolvimento de técnicas de efeito transversal a transtornos de humor variados.

As implicações da identificação de um mediador da mudança terapêutica são amplas. No contexto clínico, por exemplo, isso sugere que planos de tratamento orientados para a promoção de FP representam um caminho validado para a mudança, por meio de técnicas baseadas em aceitação (Hayes et al., 1999; 2011), mindfulness (Roemer \& Orsillo, 2009), valores (Dahl, Plumb, Stewart, \& Lundgren, 2009) e comprometimento (Kanter, Busch, \& Rusch, 2009), entre outras. Além disso,

Tabela 3. Correlações Entre FP e outros Construtos Psicológicos

\begin{tabular}{lcccc}
\hline Instrumento & Construto & Amostra & $\mathrm{n}$ & $r$ com AAQ-II \\
\hline CD-RISC 10 & resiliência & 1 & 179 & $0,35^{* *}$ \\
CES-D & depressão & 1 & 179 & $-0,62^{* *}$ \\
IDATE-T & ansiedade & 2 & 210 & $-0,71^{* *}$ \\
QSG-12 & saúde geral & 3 & 129 & $0,21^{*}$ \\
\hline
\end{tabular}

Nota. $* \mathrm{p}<0,01, * * \mathrm{p}<0,001$. 
a promoção de FP também está associada a mudanças comportamentais do clínico, como o aumento da receptividade a métodos complementares (Varra, Hayes, Roget, \& Fisher, 2008). Na área organizacional, a FP está associada ao aumento do desempenho profissional (Hayes, 2006), a maiores índices de vendas, satisfação profissional e sucesso educacional, além da redução do absenteísmo (Bond et al., 2011). Nos esportes, intervenções de promoção da FP favorecem o desempenho de enxadristas (Ruiz \& Luciano, 2009) e o aumento da força física (Secades, Terrados, García, \& Garcia, 2004). Assim, este estudo contribui ao disponibilizar uma forma de avaliação desse construto no contexto brasileiro. Isso permite o uso da flexibilidade como uma nova referência na avaliação de habilidades pessoais relevantes para a saúde e para o desempenho profissional. Além disso, também é possível usar a FP como desfecho principal em intervenções de prevenção e promoção em saúde mental, como programas de habilidades sociais ou de preparação para a aposentadoria.

No contexto da pesquisa em psicoterapia, isso significa atender a uma agenda de pesquisa antiga - e, considerando-se a proliferação de estudos sobre FP, uma agenda produtiva. Identificar um mecanismo de mudança do comportamento é uma resposta a demandas antigas, mas também apenas o princípio de outra agenda sobre os diversos contextos em que se busca a mudança comportamental.

\section{Considerações Finais}

Este estudo encontrou bons resultados psicométricos para o AAQ-II, mas limitações importantes podem comprometer a consistência das evidências empíricas. Por exemplo, a teoria que fundamenta a FP e a análise utilizada para validar o instrumento apresentam pressupostos distintos. O funcionalismo contextual pressupõe que termos como FP representam categorias funcionais de comportamento: diferentes ações que levam uma consequência comum formam uma categoria funcional. Assim, flexibilidade é apenas o rótulo de uma classe de comportamentos baseados em valores. A técnica de análise fatorial, por outro lado, assume que os itens do questionário são descrições parciais do fenômeno de interesse, que é um evento privado e não observável diretamente. Enquanto a FP é apenas o nome de uma categoria no funcionalismo, representa um fenômeno humano interno para a teoria da análise fatorial.

Além disso, há restrições metodológicas importantes. O AAQ-II é o único instrumento disponível para mensurar FP; em função da ausência de escalas e avaliações comportamentais alternativas, os resultados não podem ser comparados a medidas validadas. Instrumentos curtos são pouco sensíveis à variação, comprometendo sua aplicação para avaliar mudanças comportamentais. A homogeneidade da amostra, composta apenas por estudantes universitários, limita a generalização dos resultados para outras populações, mesmo considerando-se o grande número de participantes. Adicionalmente, apesar das evidências adequadas, a estrutura unifatorial, ainda, precisa ser submetida à análise fatorial confirmatória, assim como a avaliação das propriedades de convergência e divergência com outros construtos requerem a comparação com outros instrumentos ou a realização de análises adicionais.

Enfim, apesar de ter uma história ainda recente, as evidências empíricas sobre a FP mostram um caminho promissor, sugerindo a utilidade de estudos futuros que superem as limitações desta pesquisa e levem a um maior embasamento do construto. Considerando a abrangência do campo de estudo sobre o comportamento humano, a identificação de características que marcam transversalmente o funcionamento humano é uma contribuição significativa, principalmente, se puder desenvolver seu potencial diagnóstico e interventivo. Afinal, identificar um mecanismo de mudança pode levar a expandir a compreensão do funcionamento humano, a capacidade de influenciar o comportamento e a possibilidade de desenvolver intervenções mais precisas, econômicas e passíveis de transposição para cenários naturais diversos.

\section{Referências}

Barlow, D. H., Bullis, J. R., Comer, J. S., \& Ametaj, A. (2013). Evidence-based psychological treatments: An update and the way forward. Annual Review of Clinical Psychology, 9, 1-27.

Baron, R. M., \& Kenny, D. A. (1986). The moderator-mediator variable distinction in social psychological research: Conceptual, strategic, and statistical considerations. Journal of Personality and Social Psychology, 51(6), 1173-1182.

Bond, F. W., Hayes, S. C., Baer, R. A., Carpenter, K. M., Guenole, N., Orcutt, H. K., Waltz, T., ... (2011). Preliminary psychometric properties of the acceptance and action questionnaire-II: A revised measure of psychological inflexibility and 
experiential avoidance. Behavior Therapy, 42(4), 676-688.

Chawla, N., \& Ostafin, B. (2007). Experiential avoidance as a functional dimensional approach to psychopathology: An empirical review. Journal of Clinical Psychology, 63, 871-890.

Ciarrochi, J., Bilich, L., \& Godsell, C. (2010). Psychological flexibility as a mechanism of change in acceptance and commitment therapy. Em R. A. Baer (Ed.), Assessing mindfulness and acceptance: Illuminating the processes of change (pp. 51-76). Oakland, CA: New Harbinger.

Dahl, J., Plumb, J. C., Stewart, I., \& Lundgren, T. (2009). The art \& science of valuing in psychotherapy. Oakland, CA: New Harbinger.

Fernández, R., Secades, R., Terrados, N., García, E., \& García, J. M. (2004). Efecto de la hipnosis y de la terapia de aceptación y compromiso (ACT) en la mejora de la fuerza física en piragüistas. International Journal of Clinical and Health Psychology, 4, 481-493.

Gloster, A. T., Klotsche, J., Chaker, S., Hummel, K. V, \& Hoyer, J. (2011). Assessing psychological flexibility: What does it add above and beyond existing constructs? Psychological Assessment, 23(4), 970-82.

Hayes, S. C. (1993). Analytic goals and the varieties of scientific contextualism. Em S. C. Hayes, L. J. Reese, \& T. R. Sarbin (Eds.), Varieties of scientific contextualism (pp. 11-27). Reno: Context Press.

Hayes, S. C., \& Gifford, E. V. (1997). The trouble with language: Experiential avoidance, rules, and the nature of verbal events. Psychological Science, 8(3), 170-173.

Hayes, S. C., Luoma, J. B., Bond, F. W., Masuda, A., \& Lillis, J. (2006). Acceptance and commitment therapy: Model, processes and outcomes. Behaviour Research and Therapy, 44(1), 1-25.

Hayes, S. C., Strosahl, K., Wilson, K. G., Bissett, R. T., Pistorello, J., Polusny, M. A., Dykstra, T. A., ... (2004). Measuring experiential avoidance: A preliminary test of a working model. Psychological Record, 54, 553-578.

Hayes, S. C., Villatte, M., Levin, M., \& Hildebrandt, M. (2011). Open, aware, and active: Contextual approaches as an emerging trend in the behavioral and cognitive therapies. Annual Review of Clinical Psychology, 7, 141-68.
Hayes, S. C., Wilson, K. G., \& Barnes-Holmes, D. (2012). Contextual behavioral science: Creating a science more adequate to the challenge of human condition. Journal of Contextual Behavioral Science, 1, (1-2), 1-16.

Hayes, S. C., Barnes-Holmes, D., \& Roche, B. (Eds.). (2001). Relational frame theory: A post-skinnerian account of buman language and cognition. New York: Kluwer Academic.

Hayes, S. C., Pankey, J., Gifford, E. V., Batten, S. V., \& Quiñones, R. (2002). Acceptance and commitment therapy in experiential avoidance disorders. Em T. Patterson (Ed.), Comprehensive handbook of psychotherapy (pp. 319-351). New York: John Wiley \& Sons.

Hayes, S. C., Strosahl, K. D., \& Wilson, K. G. (1999). Acceptance and commitment therapy: An experiential approach to behavior change. New York: Guilford Press.

Hayes, S. C., Strosahl, K. D., \& Wilson, K. G. (2011). Acceptance and commitment therapy: The process and practice of mindful change. New York: Guilford Press.

Hayes, S. C., Wilson, K. G., Gifford, E. V., Follete, V. M., \& Strosahl, K. D. (1996). Experiential avoidance and behavioral disorders: A functional dimensional approach to diagnosis and treatment. Journal of Consulting and Clinical Psychology, 64(6), 1152-1168.

Hofmann, S. G., \& Asmundson, G. J. G. (2008). Acceptance and mindfulness-based therapy: New wave or old hat? Clinical Psychology Review, 28, 1-16.

Kanter, J. W., Busch, A. M., \& Rusch, L. C. (2009). Behavioral activation: Distinctive features. London: Routledge Press.

Kashdan, T. B., Barrios, V., Forsyth, J., \& Steger, M. F. (2006). Experiential avoidance as a generalized psychological vulnerability: Comparisons with coping and emotion regulation strategies. Behaviour Research and Therapy, 44(9), 1301-20.

Kashdan, T. B., \& Rottenberg, J. (2010). Psychological flexibility as a fundamental aspect of health. Clinical Psychology Review, 30(7), 865-78.

Kazdin A. E. 2000. Psychotherapy for children and adolescents: Directions for research and practice. New York: Oxford University Press.

Kazdin, A. E. (2007). Mediators and mechanisms of change in psychotherapy research. Annual Review of Clinical Psychology, 3, 1-27.

Psico-USF, Bragança Paulista, v. 20, n. 1, p. 75-85, jan./abr. 2015 
Pasquali, L. (2010). Testes referentes a construto: Teoria e modelo de construção. Em L. Pasquali (Ed.), Instrumentação psicológica: Fundamentos e prática (pp. 165-198). Porto Alegre: Artmed.

Paul, G. L. (1967). Strategy of outcome research in psychotherapy. Journal of Consulting Psychology, 31(2), 109-118.

Pennato, T., Berrocal, C., Bernini, O., \& Rivas, T. (2013). Italian version of the acceptance and action questionnaire-II (AAQ-II): Dimensionality, reliability, convergent and criterion validity. Journal of Psychopathology and Behavioral Assessment, 35(4), 552-563.

Pepper, S. C. (1966). World bypotheses: A study in evidence. Berkeley: University of California.

Purdon, C., \& Clark, D. (2001). Suppression of obsession-like thoughts in nonclinical individuals: Impact on thought frequency, appraisal and mood state. Behaviour research and therapy, 39(10), 1163-1181.

Rosen, G. M., \& Davison, G. C. (2003). Psychology should list empirically supported principles of change (ESPs) and not credential trademarked therapies or other treatment packages. Behavior Modification, 27(3), 300-312.

Rosenzweig, S. (1936). Some implicit common factors in diverse methods of psychotherapy. American Journal of Orthopsychiatry, 6, 412-415.
Ruiz, F. J. (2010). A review of acceptance and commitment therapy (ACT) empirical evidence: Correlational, experimental psychopathology, component and outcome studies. International Journal of Psychology and Psychological Therapy, 10(1), 125-162.

Ruiz, F. J., \& Luciano, C. (2009). Eficacia de la terapia de aceptación y compromiso (ACT) en la mejora del rendimiento ajedrecístico de jóvenes promesas. Psicothema, 21, 347-352.

Salters-Pedneault, K., Tull, M. T., \& Roemer, L. (2004). The role of avoidance of emotional material in the anxiety disorders. Applied and Preventive Psychology, 11(2), 95-114.

Varra, A. A., Hayes, S. C., Roget, N., \& Fisher, G. (2008). A randomized control trial examining the effect of acceptance and commitment training on clinician willingness to use evidence-based pharmacotherapy. Journal of Consulting and Clinical Psychology, 76, 449-458.

Wegner, D. M. (1994). Ironic processes of mental control. Psychological Review, 101(1), 34-52.

Recebido em: 16/12/2013

Reformulação em: 04/08/2014

Aprovado em: 09/02/2015 


\section{Anexo A}

Acceptance and Action Questionnaire-II - Versão Brasileira

\section{AAQ-II}

A seguir, você encontrará uma lista de afirmações. Por favor, avalie quanto cada afirmação é verdadeira para você e circule o número correspondente. Use a escala abaixo para fazer sua escolha.

\begin{tabular}{c|c|c|c|c|c|c}
1 & 2 & 3 & 5 & 6 & 7 \\
\hline nunca & $\begin{array}{c}\text { muito } \\
\text { raramente }\end{array}$ & raramente & $\begin{array}{c}\text { algumas } \\
\text { vezes }\end{array}$ & frequentemente & quase sempre & sempre
\end{tabular}

1. Minhas experiências e lembranças dolorosas dificultam que eu viva a vida que eu gostaria.

$\begin{array}{lllllll}1 & 2 & 3 & 4 & 5 & 6 & 7\end{array}$
2. Tenho medo dos meus sentimentos.

$\begin{array}{lllllll}1 & 2 & 3 & 4 & 5 & 6 & 7\end{array}$

3. Eu me preocupo em não conseguir controlar minhas preocupações e sentimentos.

$\begin{array}{lllllll}1 & 2 & 3 & 4 & 5 & 6 & 7\end{array}$

4. Minhas lembranças dolorosas me impedem de ter uma vida plena.

$\begin{array}{lllllll}1 & 2 & 3 & 4 & 5 & 6 & 7\end{array}$

5. Emoções causam problemas na minha vida.

$\begin{array}{lllllll}1 & 2 & 3 & 4 & 5 & 6 & 7\end{array}$

6. Parece que a maioria das pessoas lida com suas vidas melhor do que eu.

7. Preocupações atrapalham o meu sucesso.

$\begin{array}{lllllll}1 & 2 & 3 & 4 & 5 & 6 & 7\end{array}$

$\begin{array}{lllllll}1 & 2 & 3 & 4 & 5 & 6 & 7\end{array}$

\section{Sexo [F ] [M ] Idade}


Nota dos autores:

Agradecemos ao Prof. Dr. Fabio Iglesias pela colaboração na revisão da versão final do manuscrito. Os autores agradecem aos colaboradores na coleta de dados: Adriana Benevides, Ângela Lechuga, Cristineide França, Denílson Coêlho, Fabio Iglesias, Flávia Furtado, Gean Carlos, Gustavo Tozzi, Hilma Khouri, Juliany Guimarães, Marianna Braga, Mathieu Turgeon, Marck Torres, Michaela Saban, Patrícia Ribeiro, Solange Alfinito e Zenith Delabrida.

Sobre os autores:

Leonardo Martins Barbosa é psicólogo, mestre e doutorando em Psicologia Clínica pela Universidade de Brasília, na qual estuda prevenção e promoção em saúde mental aplicada à preparação para a aposentadoria.

E-mail: leopfq@gmail.com

Sheila Giardini Murta é professora adjunta no departamento de Psicologia Clínica da Universidade de Brasília $(\mathrm{UnB})$, orientadora no programa de pós-graduação em Psicologia Clínica e Cultura (UnB), realizando pesquisas em prevenção e promoção em saúde mental, e bolsista de produtividade em Pesquisa - CNPq. E-mail: giardini@unb.br

Contato com os autores:

Departamento de Psicologia Clínica, Instituto de Psicologia, Universidade de Brasília Campus Darcy Ribeiro, Brasília, DF

CEP: 70910-900

E-mail: leopfq@gmail.com 